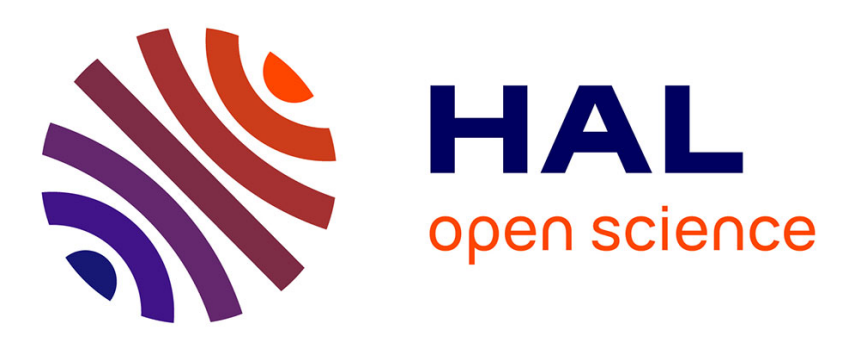

\title{
Figuration de l'intraduisible de l'exclusion à travers le dispositif de supervision
}

Derek Humphreys

\section{To cite this version:}

Derek Humphreys. Figuration de l'intraduisible de l'exclusion à travers le dispositif de supervision. Cliniques méditerranéennes, 2014, L'intraduisible, la langue et le lien social, 90, pp.103-116. 10.3917/cm.090.0103 . hal-02523959

\section{HAL Id: hal-02523959 \\ https://hal.science/hal-02523959}

Submitted on 30 Mar 2020

HAL is a multi-disciplinary open access archive for the deposit and dissemination of scientific research documents, whether they are published or not. The documents may come from teaching and research institutions in France or abroad, or from public or private research centers.
L'archive ouverte pluridisciplinaire HAL, est destinée au dépôt et à la diffusion de documents scientifiques de niveau recherche, publiés ou non, émanant des établissements d'enseignement et de recherche français ou étrangers, des laboratoires publics ou privés. 
Le dispositif de la supervision et figuration du terrain intraduisible de l'exclusion.

La fonction de traduction dans l'intervention psycho-sociale auprès des immigrants en situation d'exclusion.

Supervision and figuration of the untranslatable field of exclusion.

The translation function in psycho-social intervention with homeless immigrants

HUMPHREYS Derek. Psychanalyste, Maître de Conférences

Aix Marseille Université, LPCLS EA 3278, 13621 Aix-en-Provence, France

Adresse : $\quad$ Aix Marseille Université

Laboratoire de Psychopathologie Clinique, Langage et Subjectivité (LPCLS EA 3278)

29 Av. Robert Schuman

13621 Aix-en-Provence Cedex 1, France 
Le présent article se base sur une expérience de travail avec des personnes immigrées vivant dans la grande précarité. Travail qui nous confronte à trois points critiques. Tout d'abord, l'exclusion et l'absence de lien social dans le pays d'arrivée. En effet, le processus de déliaison associé à l'exclusion est encore plus grave quand le sujet disparait non seulement dans le regard mais aussi dans la langue -la plupart des immigrés dans la précarité balbutie quelques mots de français mais leur enfermement se voit conforté par leur repli sur la langue maternelle. La barrière de la langue constitue ainsi le deuxième point de butée de ce travail une langue qui sert d'enveloppe et qu'isole le sujet, loin du réel de la rue, dans un ailleurshors-temps. S'ajoute à cette double exclusion -de l'incompréhension et l'acculturation- le trauma, troisième aspect critique. Le trauma qui a éloigné ces personnes de leur pays et leur famille et qui s'actualise chaque jour dans la désocialisation. Nous touchons ainsi le paradoxe de ce travail, qui donne accès -dans le meilleur des cas- au roc de la déliaison pulsionnelle, de l'anéantissement subjectif et de l'exclusion sociale seulement après un long parcours d'élaboration de liens fiables, de reconnaissance singulière et subjective, dans une langue actualisée ici, dans un écart temporel qui pourrait l'élaborer sous la forme d'un récit.

Les cliniciens travaillant dans ce terrain voient leur pratique depuis quelques années se dissoudre dans les segmentarisations administratives et politiques, quelque part entre le médico-psychologique et le psycho-social. Au carrefour du psychopathologique et du social, l'individuel et le collectif, les initiatives d'intervention dans ce domaine se voient contraintes à s'adapter aux référentiels médicaux ou sociaux en circonstances que l'expérience a démontré que les enjeux psychiques qui font résistance au changement demeurent intouchés (Douville 2000). Dans ce travail, il ne suffit pas de soigner ou de chercher une intégration sociale. Il faut intervenir ailleurs dans la mesure où ce qui se délite n'est pas la capacité à se soigner mais la capacité à construire et entretenir des liens -au-delà de l'anonymat du foyer social, il faut un TU qui nomme et subjectivise. 
A partir de la supervision de cliniciens travaillant sur le terrain de la précarité et de l'exclusion des immigrants d'Europe de l'est, cet article articule la psychopathologie au social dans l'accès au trauma et aux conditions de mise en récit du périple singulier de survie de chacun de ces échappés de l'horreur qui viennent en trouver un autre sur les trottoirs de la ville. Tout en présentant les quelques bribes d'histoire qui ont donné accès à des récits possibles, est proposée une approche métapsychologique au travail des équipes mobiles d'accompagnement à ces sujets. Est discutée la particularité des dispositifs crées dans les interstices institutionnels par ces équipes, qui vient répondre à la nécessité d'un espace et d'un temps d'accueil spécifiques, en adéquation à un cadre à la fois «portable », plastique et contenant (Roussillon). Capable d'établir des liens fiables et de donner une place à l'étant dans ce contexte d'exclusion et d'anéantissement.

Cette analyse s'intéresse spécifiquement à l'élaboration dans l'après-coup de la rencontre avec ces personnes -tant elle demeure incertaine. Face à l'excès de réel d'une rencontre dans l'actualité du trottoir, la place pour un sujet ne peut surgir que dans l'ailleurs de la supervision et dans la capacité de ces professionnels à l'inscrire affectivement et subjectivement (Beetschen), en construisant un lien qui passe par le biais du besoin tout en visant à donner une place au sujet et à son désir.

\section{$\underline{\text { Etrangers dans cette langue }}$}

Le sujet se constitue dans un rapport nécessaire et permanent aux autres, dans un allerretour constant entre des mouvements individuels de signification de l'environnement et des échanges avec d'autres qui donnent une valeur symbolique à cette signification. L'Autre, qui donne accès à la représentation, demeure ainsi fondamentalement lié à l'objet.

Ce rapport complexe individu-société s'organise aussi bien au niveau des mécanismes sociaux que des dynamiques psychiques et des liens intersubjectifs, groupaux ou culturels. 
Toute appartenance groupale étant établie par un mode relationnel, elle permet une reconnaissance et par cette voie une identité -les processus d'intégration et d'exclusion sociale sont en effet constitutifs de l'individu (Stitou). Ces modes relationnels organisent aussi des modes de manifestation de l'affect, de construction d'une mémoire, d'élaboration du trauma. En effet, si le sujet s'inscrit dans le discours par le lien social, le symptôme est souvent la manifestation de l'assujettissement du sujet au discours. Aussi, un groupe ou une société peut passer par une expérience pour laquelle il n’y a ni construction de mémoire ni signification du trauma, affectant la relation du groupe à l'individu -sur le plan de la reconnaissance subjective et symbolique ainsi que celle des significations culturelles du nonréalisé-non-symbolisable.

Dans le travail avec des immigrants en situation de précarité, nous sommes confrontés à une double rupture. A celle des liens entre le sujet et l'Autre, qui se manifeste par une perte de désir et d'identité (Douville 2004) vient s'ajouter le mouvement migratoire, redoublant. On voit ainsi échouer sur les trottoirs de Paris des hommes et des femmes ayant échappé la persécution ethnique en Ouzbékistan, ayant perdu des repères identitaires en Lituanie, se sentant exclus de l'espace urbain en Pologne, ne trouvant plus d'éléments sociaux de reconnaissance en Russie...

Leurs expériences précoces ou répétitives ayant démontré que l'objet désiré n’est jamais trouvable dans l'environnement, ces sujets n'espèrent rien des liens et désinvestissent les objets du monde. La perte de confiance dans le lien, irrémédiablement précaire, et le caractère insupportablement éphémère des objets explique leur isolement et leur attachement à un petit ensemble de choses ayant démontré une utilité : une gamelle, une couverture.

L'actualité du besoin qu'impose la survie dans la rue rend impossible toute projection dans le fantasme névrotique d'une vie meilleure, toute volonté d'intégration, tout projet de vie. Effaçant leur histoire et écrasant toute possibilité de désirer. La rue et la précarité 
constituent ainsi des solutions : coupés des modes habituels d'échange, leur solution de survie passe par l'occupation des interstices du social. Or, les discours sociaux gomment toute solution subjective et imposent des interventions qui font l'impasse sur le sujet et sa parole (Berger).

Fournir un toit et de la nourriture sont des réponses appropriées mais insuffisantes pour ces sujets, qui refusent la normativité des échanges sociaux dont ils sont exclus. Cette association d'abandon physique et misère psychique relève d'une approche métapsychologique (Jacobi) capable de tenir compte des aspects individuels et collectifs du trauma et d'une clinique des liens entre l'individu et le groupe en situation extrême capable d'articuler l'opposition que trouve le corps social, qui s'organise en termes de dedans/dehors, public/privé, face à une souffrance individuelle qui occupe des interstices et échappe toujours aux segmentarités (Pommier 2003). Cette approche n'envisage pas l'absence de représentation collective et subjective dans sa négativité, mais comme un agir susceptible d'être élaboré. Les interstices symbolisants peuvent être créés dans ce même matériau, sur lequel des allers-retours du dehors au dedans, du singulier au collectif, du public au privé sont possibles, dans une démarche capable d'historiciser l'expérience traumatique individuelle associée à l'exclusion (Benhaïm).

\section{$\underline{\text { Interprètes d'un cadre intraduisible }}$}

Dimitri, 55 ans, toxicomane sous traitement de substitution, séropositif au VIH et à l'hépatite C, a essayé de braquer une banque à son arrivée en France avec le groupe d'amis qui avait émigré avec lui. Après avoir purgé sa peine pendant 2 ans il commence à fréquenter les lieux d'accueil où il a pu rencontrer l'équipe Bociek ${ }^{1}$. Il a été reçu régulièrement par le

\footnotetext{
${ }^{1}$ Bociek est une équipe mobile d'intervention psycho-sociale. Organisé en binômes psychologue-assistant social, ils travaillent auprès d'immigrants russes (ou russophones), polonais, bulgares et roumains.
} 
binôme russophone de l'équipe pendant 7 ans. Dans une confusion délirante et très agressive, les premières années du suivi furent marquées par la peur des thérapeutes : il envoyait par SMS des images de lui, torse nue, montrant son corps tatoué et scarifié, entouré d'un halo rose et une musique stridente -réminiscence maternelle crue et persécutrice. Il passait aussi par des périodes où il ne supportait pas la présence de l'une de ces professionnelles dont il se sentait désiré et qui lui faisait des avances, le recevant déshabillée dans son bureau. Il y avait toujours l'autre pour l'entendre et apaiser ses angoisses. Il a raconté pendant 3 ans l'histoire de la femme qui a profité de lui à son arrivée en France et qui a essayé de le faire déporter dans son pays, avant de pouvoir accéder à un premier récit familial. Dans celui-ci, il est le fils d'un fonctionnaire russe haut placé que sa mère Géorgienne a accepté en mariage pour avoir accès à un certain confort. Le nœud du récit se fait à 8 ans, moment, où son père l'aurait envoyé en camp de redressement militaire, où sa mère l'abandonne, où il rencontre un monde violent et abusif qui l'amène vers une série de séjours en prison, la persécution par l'état, etc. Ce récit d'aspect psychotique ouvre des interrogations psychopathologiques mais la continuité du travail se fait surtout dans l'élaboration de ce contre-transfert inquiétant et persécuteur, truffé de menaces et de sexuel primaire. Ayant passé les derniers 4 ans dans un foyer et accompagné régulièrement par le binôme russophone, sur lequel il a pu organiser ses représentations du bon et du mauvais objet -et un nouveau rapport au féminin- le délire a disparu depuis un an. Il comprend que des liens sociaux d'amitié et, ou de famille le protègent des femmes qui pourraient le désirer. Il a humanisé le récit de sa mère et son roman familial semble moins violent et moins persécuteur aujourd'hui -bien que les éléments traumatiques et la perte de racines et de liens soient toujours là.

D'autres sujets dans l'exclusion ne s'en sortent pas aussi bien. Deux des psychologues de Bociek ont dû se rendre aux tunnels qui traversent les halles de Paris au moment de leur fermeture car les structures sociales de la ville voulaient recenser la population qui occupait 
cet espace. Ils ont trouvé ainsi une jeune polonaise toxicomane qu'ils avaient pu rencontrer 3 ans auparavant, à son arrivée à Paris -moment où elle commençait à décrocher des liens, tout en cherchant encore une issue. Lors de cette visite aux tunnels ils ont trouvé une population complètement scotchée au bitume, vivant dans l'ombre, entre les pots d'échappement. Et, cette même jeune femme enfermée jour et nuit dans une tente, partageant avec les autres et avec les rats un pot de restes de nourriture posé à même le sol, dans ce petit espace entre le mur du tunnel et la voie des voitures. C'est avec cette vision d'une désorganisation subjective des humains devenus des détritus posés là où rien ni personne ne s'arrête qu'il faut souvent envisager une présence capable de proposer un matériau d'intrication à cette déliaison mortifère (Pommier 2008).

Ce qui caractérise le trauma est son incapacité à faire représentation. C'est ce qui le fait demeurer hors psyché, corps étranger qui fait effraction dans le système des représentations. Le trauma lié à la migration et aux effets inconscients et trans-générationnels provoqués par les circonstances de ces mouvements -dictatures, guerres, persécution ethnique- en tant qu'évènement jamais reconnu, fait retour dans l'impossibilité de construire des liens. Cette précarité du lien entraine à son tour une décomposition identitaire et une succession de violences internes (pulsionnelles) et externes (sociales). Cette absence de représentation collective et subjective, envisagée comme une manière d'agir le trauma par la coupure et la perte de liens, constitue notre matériau de travail.

Sur le terrain de la non-symbolisation, dans la recherche de mémoire face à des situations extrêmes, ces sujets ont créé une solution dans une forme particulière d'occupation de l'espace (public/privé, intérieur/extérieur). Ces actualisations constituent le matériau d'une transformation de déplacement ou de mise en scène d'un trauma qui ne fait pas mémoire ni histoire et qui semble caractériser la psychopathologie de l'errance mais s'inscrit autrement dans cette situation d'exclusion dans la langue et d'appel à la traduction. Ces matériaux sans 
représentation peuvent en effet servir de socle aux processus psychiques de transformation processus originaires donnant accès au fantasme (Aulagnier, p.82-91)- dans l'élaboration des traductions successives du non-symbolique-non-représenté, dans son articulation du singulier du trauma aux difficultés d'un peuple à des moments historiques qui empêchent le processus de übertragung -traduction/transport/transfert- créant des fueros au fonctionnement psychique (Freud 1896, p.155). Ce premier appel à la traduction/transfert est ici étroitement associé à la rencontre avec un autre dans une langue qui associe la traduction au transfert dans un mouvement de régrédience (Botella) qui restitue la charge affective et figurative aux mots.

Nous sommes, certes, tous des étrangers dans la langue. Si l'inconscient -qui pourtant le détermine- demeure à jamais inaccessible au sujet, la rencontre avec les autres et l'Autre signe à son tour cette étrangeté et cette étrangéité dans la préexistence du langage. La langue, déjà là, organise le lien du sujet aux autres. D'un sujet devenu étranger à lui même en découvrant qu'il existait déjà dans la langue et qui rencontrera de surcroit, dans ce rapport nécessaire à la langue, le manque à tout dire : les mots sont toujours insuffisants et tout énoncé devient ainsi un malentendu. L'étranger est «la base du lien social... au cœur du langage », affirme Rajaa Stitou (p.198). Cette étrangeté est intraduisible et touche chaque sujet de la même manière, dans toutes les langues. Or ce sont bien des interprètes qui interviennent dans la coupure des immigrants en situation d'exclusion, qui apportent avec cette langue partagée une possibilité de lien. Comme si après la séparation et la perte de tout, les sons d'une langue infantile, archaïque, au delà (ou en deçà) du sens, s'avérait capable d'intriquer le mot à l'affect, constituant un mouvement de symbolisation et de reconnaissance.

Envisageant la rue et la précarité comme solutions, Benhaïm (2012) propose un travail fait dans l'alternance entre les espaces de soins et de parole pour « faire avec l'(A)utre » et inscrire dans ce lien au socius les fragments d'une vie effacée. Apaisant le souvenir, transformant le trauma en épreuve. Ce travail fait dans l'entre-deux profite des interstices 
paradoxaux, par la médiation d'un impératif administratif de réinsertion sociale, dans la temporalité du mandat politique, mais dans une déliaison -sociale et singulière- qui exclut toute soumission aux segmentarités. Les professionnels se proposent ainsi comme interprètes dans un accompagnement dans l'accès au droit aux soins, dans la rencontre avec le médecin ou l'assistant social pour l'accès à un toit provisoire. Cette offre d'accompagnement et de traduction ne peut en effet être entendue comme un pur passage d'une langue à une autre, car le sujet en situation d'exclusion est foncièrement coupé du lien social. La traduction est ici possible par une première reconnaissance dans la langue qui fait transfert, en renouant avec le lien -de manière certes precaire- pour ces sujets exclus. La présence régulière mais informelle des intervenants de l'équipe constitue ainsi une première adresse à un autre qui leur donne existence, dans l'étrangéité de leur propre langue. Tout à coup, quelqu'un reconnaît leurs expressions, entend leurs cris -ces sujets hurlent leur savoir sur le manque (Jacobi)- et ressent la violence et le poids des mots. En les écoutant, cet autre les interpelle, les ancre à la Langue après une longue traversée du désert. Les rencontres successives avec celui qui traduira les maux pour le médecin, qui remplira avec eux des dossiers et qui les retrouvera toujours au même repère de leur déambulation sans destin, les amène à parler d'une autre ville, d'une famille, d'un ailleurs-autrefois. De persécutions politiques, de violences ethniques, d'abus, de trahison.

La présence d'un humain capable de donner une réponse en adéquation au besoin Nebenmensch- donne accès au registre du désir. Le sujet advient ainsi dans le mouvement de quête d'une retrouvaille avec cet autre qu'en répondant au besoin apporte une enveloppe sensorial et une nomination (Freud 1895, p.441). Le mouvement pulsionnel sort du registre purement énergétique dans cette rencontre avec l'Autre de la nomination et la représentation. Or le sujet ayant connu précocement la perte, ayant fait l'expérience d'un Nebenmensch élusif 
et fragile et d'un Autre persécuteur et violent, n'a pas fait trace de ce mouvement. L'illusion d'un lien fiable ne fut pas possible.

Winnicott nuance ce passage du besoin au désir : la capacité à être durablement là de l'autre, à supporter la violence et la destructivité, fait de l'objet créé-trouvé un objet d'attention et un lien de concern (Winnicott, p.74). La capacité de symbolisation de cette présence constitue une exigence énorme, l'engagement du corps propre dans le tissage d'un espace transitionnel (Benhaïm).

On comprend l'importance du lien établi entre les sujets dans l'exclusion et un autre disponible : il inaugure la possibilité de mettre à l'épreuve la contenance de l'environnement, la disponibilité de l'objet, la fiabilité. La disponibilité de ceux qui répondent aux besoins primordiaux de survie crée les conditions de régression qui donnent accès à la symbolisation et à l'affect -la capacité de concern dépend de la disponibilité et de la capacité de survie de l'objet. Dans ce travail, qui se fait dans l'échange entre deux adultes, ceci passe par l'établissement d'une fonction de bord, dans le sens où l'adulte qui se rend affectivement disponible sert de rive à un mouvement fait vers l'extérieur quand il devient possible de croire en la satisfaction. Mouvement pulsionnel qui accepte l'illusion d'une satisfaction, qui accepte une représentation d'objet et peut le désirer. Qui pourra établir le lien qui noue le Moi à celui qu'apporte un Fremde Hilfe -qui sort le sujet de l'état de Hilflosigkeit (Freud 1895, p. 411).

Ce travail qui s'offre comme bord dans les interstices pour la constitution d'un mouvement pulsionnel de signification désirante prend compte des processus psychiques qui caractérisent 1'exclusion. La fonction de bord qui fait lien par une présence réelle déplace ainsi le sens même du social et du discours.

La condition pour s'offrir comme bord aux mouvements d'un sujet dans la déliaison de la précarité passe certes par un désir d'être là qu'il faut suivre de près pour en faire un outil de travail. Il serait impossible de développer dans ces quelques lignes la complexe question de 
ce désir. Cet article se limitera à l'analyse des possibilités d'élaboration de celui qui s'offre comme point d'ancrage pour un sujet en déambulation.

\section{$\underline{\text { Supervision et figuration }}$}

L'espace de la supervision permet de penser l'accès à la figurabilité dans ce terrain où des lambeaux de jouissance poussent à l'ombre de la parole et ne se manifestent que dans le transfert qui -hors cure- requiert d'un dispositif spécifique pour être élaboré. En termes foucaldiens, le dispositif est une relation du savoir à la praxis capable de mettre au travail de manière créative et innovante -par opposition à la prédictibilité du structuraliste- des éléments propres à une population, un temps, une architecture, des codes sociaux (Agamben). Situation qui concerne pour nous le sujet immigrant isolé ou en petit groupe vivant à la rue, sans famille, racines ou histoire. Des sujets qui, ayant échappé à la violence ethnique ou politique, ayant perdu tout lien avec une tradition culturelle après des décennies de régime soviétique, déambulent dans les rues de Paris, non pas à la recherche de quelque chose mais échappant à une langue et à une société qui n'inscrit rien de leur histoire. Espérant en construire une dans cette distance et cette étrangéité qui pourraient leur permettre l'abandon de soi mais qui finit par les rendre invisibles, les anéantir et les voir mourir de froid à Stalingrad. Ce dispositif dépend de la distance que chacun arrive à établir à l'égard d'une certaine construction de la clinique et son rapport à une position éthique. Distance qui donne accès à l'écoute du roc de ces situations extrêmes -qui ne sauraient pas être expliquées par la seule référence psychopathologique. Le but serait plutôt de rendre sensible, figurable, le conflit qui sous-tend ce qui est donné à voir dans sa relation aux éléments politiques, urbains, architecturaux, fantasmatiques.

Dans ce travail proche de la déshumanisation, la subjectivité ne saurait pas être retissée dans l'immédiateté du réel, sans demande et sans transfert (Jacobi, p.96). Elle exige 
que le mouvement soit inscrit ailleurs, déplacé dans le temps et l'espace. Cette distance qui reproduit ailleurs le mouvement entre deux sujets, donne place à un souffle. Inspiration musicalement un silence et un rythme- qui signale un bord, qui délimite des corps habités. Puis qui permet une écriture -substantialité du rythme de ces échanges- et une adresse dans laquelle on se raconte -récit qui fait place aussi bien au mythe qu'à l'histoire d'une famille, d'un peuple, d'une perte, d'une tradition. Transféré sur le dispositif de la supervision, dans le récit se déplient des parcours, des subjectivités, des matériaux historiques, biographiques, géographiques qui prennent forme dans ce souffle pour signifier subjectivement l'expérience douloureuse de la perte de famille, du pays, de l'identité. L'excès de réel -la faim, le froid, les corps en souffrance- plus qu'une flexibilité du cadre, exige une figuration des liens dans l'après-coup de la supervision, loin de l'actualité de la rue. Dans l'immédiat de la rencontre est à l'œuvre la contingence de la précarité mais aussi celle des intervenants. De surcroit, quand cette rencontre se fait dans une langue qui sort l'un et l'autre de l'actuel du trottoir sans représentation- en les renvoyant dans un ailleurs-autrefois -avec toute sa charge de bienveillance, de frustration, d'étonnement et de rejet.

Ce travail in presentia ne peut pas se faire dans les segmentarités de l'institution, des espaces clos, des temps de séance. Il se fait dans un temps suspendu, dans les interstices et des lieux de circulation. Là où le sujet ne se reconnaît pas dans un statut bien délimité, dans une adresse encadrée. Dans les interstices, en dehors du cadre social, l'étonnement inscrit une présence d'emblé dans la sensorialité. Sur le terrain de la grande précarité, les interstices sont démultipliés par l'état, par la société, qu'imposent un système d'organisation, de recensement, d'effacement par normativation. Les intervenants sont contraints à trouver une place entre le médico-psychologique et le psycho-social, de médicaliser par le diagnostic quand on sort du cas social. L'adresse ne peut se faire qu'autour d'une demande de logement ou de médication. L'accueil se fait ainsi dans cet interstice politique qui n'est ni médico-psychologique, ni 
psycho-social, mais dans la rue, dans des temps de déambulation et de passage, toujours entre deux. Au carrefour, donc, du psychique et du social, de l'individuel et du collectif, de la construction identitaire et ses liens avec la construction civilisatrice. Cette adresse apparaît ainsi comme la seule possibilité d'exister, d'abord, puis de figurer le vide, l'effacement, dans la recherche d'une surface capable de faire bord. Or cette reconnaissance se fait dans l'étrangéité de la langue. Ainsi, l'offre d'un accompagnement d'interprète dans les démarches administratives d'accès aux papiers, aux soins, éventuellement à un toit sert d'abord à répondre aux logiques administratives d'intégration et à une exigence de comptabilité des interventions -satisfaction, suivi. L'expérience montre que cette occupation des interstices, qui permet de répondre à une demande matérielle, est l'alibi à une relation qui redémarre la machine désirante et qui permet de mettre à l'épreuve la fiabilité du lien : une fois le logement ou les papiers obtenus le sujet ne se présente pas le jour de la remise mais réapparait quelques semaines plus tard, pour renouer son lien au psychologue ou au travailleur social.

La création plastique -individuelle ou plurielle- qu'inscrit cet «entre-deux », le mouvement adressé à un autre qui fait bord, constitue un nœud qui lie le singulier au collectif dans le millefeuille culturel. Cette inscription n'est possible que quand une surface se prête à la création. La supervision est un espace qui s'offre comme matériau d'inscription et de traduction/transfert (übertragung). Le pli dans l'espace et le temps qu'offre la supervision détermine une adresse et donne ainsi lieu à un récit de ce qui était resté une pure expérience dans une langue infantile, entre un sujet coupé du social et celui qui interprète son isolement. Ce dispositif contenant des mouvements transférentiels donne ainsi accès à une Darstellung (David-Ménard) du traumatique.

Sortant de la pure négativité du traumatique-non-symbolisé, la notion de Darstellung (figurabilité) nous permet d'élaborer l'accès aux mots de ces modes de l'agir liés au trauma de l'exclusion. L'absence de représentation collective et subjective du lien, sans ce dispositif 
d'adresse qui fait résonnance aux échanges entre le sujet en situation de précarité et cet autre qui se propose comme objet à utiliser, ferait impasse dans la pure décharge du trop d'intensité de ces échanges dans un procédé protecteur individuel. Le récit de ces derniers dans le dispositif -transférentiel- de la supervision fait inscription et figure, par résonnance, en saisissant les matériaux de l'expérience de ces échanges dans leur substantialité -musicale ou poétique, dans l'intraduisible de cette langue infantile archaïque. Les figures, hors représentation, s'inscrivent à même le corps. C'est sur ce passage de la sensation à la perception que le clinicien est amené à intervenir, dans la possibilité d'inscrire le parcours d'une ville ou même d'un foyer d'hébergement d'urgence, dans l'appropriation d'une image du corps qui organise une enveloppe capable de faire socle à la construction d'une histoire personnelle. Dans leur qualité picturale, tonale ou poétique, dans leur dissonance ou leur accord rythmique. L'appropriation dans le récit de ces éléments perceptuels donne accès à la représentation de l'autre, de soi et d'un Autre. Cet ensemble de représentations pourra amener à son tour à une image identitaire susceptible d'être narcissiquement investie. Loin des voies cognitives ou représentationnelles classiques, la capacité créatrice de la mise en scène de l'horreur de la dé-subjectivation et de l'odeur de la déshumanisation est transformée par déplacement dans cet espace contenant.

Le récit apporté à la supervision retrace ainsi les effets d'un mouvement énergétique inscrit dans l'actualité du corps et de la scène -des sensations olfactives, des réactions visuelles et tactiles- qui présentifie le trauma et la rupture. La décharge énergétique sert à éviter une symbolisation impossible, non-réalisée. L'entre-deux de la rencontre, en reconnaissant la qualité de quelle, de source pulsionnelle du corps du sujet en situation d'exclusion par la position même d'un autre contenant, fait trace. Le corps réel dé-subjectivé affecte ainsi, dans toute son étrangeté - unheimlich- un autre capable d'introduire l'après-coup dans son récit. Il va de soi que la condition de cette présentification est la présence, face à ce 
corps réel, d'un autre susceptible d'être affecté en creux. Capable de voir, en deçà des catégorisations sociales, des constructions métaphoriques et du décryptage médical. Dans la spécificité de sa mise en scène fantasmatique : si l'objet de la pulsion est un tenant-lieu du vide et le sujet est une vacillation entre deux signifiants, cette surface à double bande qui fait entre-deux inscrit la trace des mouvements -dans une mémoire sensorielle- pour faire inscription, parole et représentation dans le champ de l'Autre.

\section{Conclusion}

Nous sommes tous confrontés, au quotidien, à l'exclusion, l'errance, la précarité dans notre rapport à l'espace public. Les processus subjectifs touchant la question de l'intégrationexclusion ne dépendent pas seulement de l'intimité psychique ou des liens établis précocement dans le processus de subjectivation mais s'installent également dans les espaces collectifs, prenant des formes plus ou moins visibles. Chaque parcours subjectif se voit aussi traversé par les espaces publics quotidiens où il se déroule. Certaines situations extrêmes de dé-subjectivation font de l'espace public un terrain d'organisation de ces conditions extrêmes, par exemple dans la constriction de trajectoires psychosociales alternatives.

Dans ces quelques lignes, nous avons envisagé l'exclusion comme un phénomène qui ne concerne pas uniquement l'économie, mais aussi la psychologie, la médecine, la politique et la culture : les stratégies destinées uniquement à répondre aux besoins des individus en situation de précarité (somatiques, sociaux, d'hébergement) se sont avérées insuffisantes et montrent l'importance d'une convergence entre le social et l'individuel.

L'expérience discutée dans cet article, dans l'actuel du besoin et de la survie, accompagnant au mieux une soupe qui réchauffe le corps -et plus souvent le crachat repoussant, une odeur pénétrante, une misère effrayante- exige d'y aller, sortir de soi et des hypothèses de travail pour intervenir dans le réel. La présence est réel. Elle s'offre comme 
bord pour inscrire un mouvement qui s'adresse à l'extérieur et qui fait résonnance, produit (un) rythme, donne une continuité, établit une poétique dans l'entre-deux. C'est grâce, à la fonction de bord que le thérapeute peut inscrire sa capacité à reconnaître l'autre, que le spéculaire peut renvoyer ces individus hors lien vers une existence désirante qui fait représentation et qui peut avoir une certaine fiabilité. Nous ouvrons avec cette réflexion sur les processus qui permettent de rendre présent un objet encore inexistant -au sens où l'entend Fédida (1995), en tant que non-inscrit et imperceptible- la brèche qui sépare le registre du refoulé -interprétable- de celui de l'agir traumatique -qui n'appelle pas d'interprétation, qui demeure hors champ de la parole en pensant.

Le trauma est cependant susceptible de faire retour, faire symptôme dans la constellation signifiante apporté par cet autre qui fait bord au mouvement pulsionnel. Dans cet entre-deux, interstice social, aller-retour entre le public et le privé, entre le dedans et le dehors, nous trouvons le matériau dans lequel se fait le mouvement symbolisant. C'est à partir de cette expérience singulière qu'on peut aussi passer du récit de l'horreur individuelle, à la mémoire universelle, à l'historisation du trauma dans une recherche de mémoire et de reconnaissance.

Nous avons proposé que l'inscription subjective de cette expérience partagée qui fait bord aux mouvements pulsionnels et constitutifs de l'altérité, ne puisse se faire que dans une autre scène. Le récit subjectif de ces rencontres en supervision, dessine ces mouvements dans la résonance des signifiants qui constituent le matériau d'une musicalité, d'une chorographie d'échanges, sans les inscrire, nécessairement, dans un lien direct aux expériences infantiles de chacun. Le groupe sert de contenant à ces matériaux qu'il situe ailleurs, ni dans le pays d'origine, ni dans l'actualité de cette autre ville, faisant à son tour fonction de bord face aux moments de crise où chacun s'approche des limites de sa propre capacité de figurer l'autre en lui. 


\section{Bibliographie.}

AGAMBEN, G. 2007. Qu'est-ce qu'un dispositif ? Paris, Rivages.

AULAGNIER, P. 1975. La violence de l'interprétation, Paris, PUF.

BEETSCHEN, A. 2008. «Sur le maniement de la supervision », dans Topique 103, 31-40.

BENHAIM, M. 2012. «Vivre est plus difficile que survivre », dans Clinique psychanalytique de l'exclusion (sous la direction de O. Douville), Paris, Dunod, 137-164.

BERGER, F. et coll. 2008. "Clinique du sujet et du lien social contemporain », dans Cliniques Méditerranéennes, 78(2), 83-98.

BOTELLA, C. 2001. «Figurabilité et régrédience », dans Revue Française de Psychanalyse $65,1149-1239$

DAVID-MENARD, M. 1983. L'hystérique entre Freud et Lacan. Corps et langage en psychanalyse, Paris, Editions Universitaires.

DOUVILLE, O. 2004. "Anthropologie du contemporain et clinique du sujet », dans L'évolution psychiatrique, 69(1), 31-47.

DOUVILLE, O. 2000. «Pour introduire l'idée d'une mélancolisation du lien social », dans Cliniques Méditerranéennes, 63, 239-261.

FEDIDA, P. 1995. Le site de l'étranger, Paris, PUF.

FREUD, S. 1896. «Lettres à Fliess (No.52)», dans La naissance de la psychanalyse, Paris, PUF (1956), 153-160.

FREUD, S. 1895. «Entwurf einer Psychologie », dans Gesammelte werke, Nachtragsband, Frankfurt am main, Fisher (1987)

JACOBI, B. 2005. «Précarité psychique, lien social », dans Cliniques Méditerranéennes, 72 (2), 89-102.

POMMIER, F. 2008. L'extrême en psychanalyse, Paris, Campagne Première.

POMMIER, F. 2003. « Situaçaoes extremas e figuras do informe », dans Revista Latinoamericana de psicopatologia fundamental, 6(1), 86-99.

ROUSSILLON, R. 1997. "L'errance identitaire», dans Souffrance psychique, contexte social et exclusion, actes du colloque de Lyon Bron, ORSPERE.

STITOU, R. 2012. «L'extimité de l'étranger », dans Cliniques Méditerranéennes, 86(2), 197 207.

WINNICOTT, DW. 1963. «The development of the capacity for concern », dans The maturational process and the facilitating environment, London, Karnak (1990), 73-82. 


\section{$\underline{\text { Résumé }}$}

Le présent article propose une élaboration métapsychologique de la construction du lien individuel et de l'inscription sociale à partir d'un travail de supervision d'une équipe d'intervention psycho-sociale auprès d'immigrants de l'Europe de l'est en situation de précarité à Paris. L'écart temporel et spatial dans ce travail paraît nécessaire face au poids de l'actuel de la rue, ainsi qu'à l'importance de ce qui est fait in presentia et qui met l'un et l'autre, le sujet en situation de précarité et l'intervenant, en situation de régression du fait de ce décalage produit par la rencontre dans la langue. Le travail de figurabilité de l'intraduisible de ces mots partagés est proposé à l'intérieur du dispositif, comme une instance capable de renouer perception et langage, sortant ainsi le sujet de la sidération traumatique dans un récit devenu possible à partir de la figuration de la rencontre par cet autre qui réactualise la possibilité d'un lien fiable.

Mots-clés : Précarité - Exclusion - Lien Psychique - Figurabilité - Supervision - Immigrants

\section{$\underline{\text { Abstract }}$}

Based on the clinical supervision of a psychosocial intervention team working with easternEurope immigrants in a precarious situation in Paris, the present article proposes a metapsychologial elaboration of the development of subjective boundaries and of a possible social inscription of this population. A temporal and spacial gap seems necessary to this elaboration, specially regarding the weight supported by the actual experience of the street and the importance of what needs to be done in presentia and that puts both, the subject in precarious situation and the person who comes to his encounter, in a regressive situation. This latter is due to the shift induced by the shared language -so close and yet so distant for both of them in this given situation. The process of figurability of these untranslatable shared words becomes possible within the supervision, renewing the perception-language continuity and offering the subject a way-out from the traumatic sideration through a narration that starts with the figuration of this encounter with this other who updates the possibility of a reliable boundary.

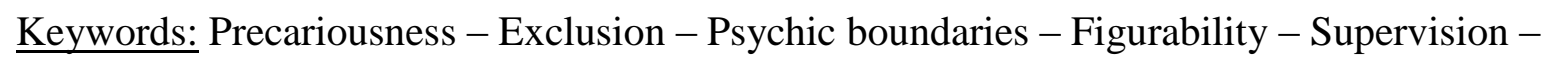
Immigrants 